\title{
Sizes and interactions of halo nuclei
}

\author{
J.A. Tostevin, J.S. Al-Khalili, J.M. Brooke, and J.A. Christley ${ }^{1}$ \\ ${ }^{1}$ Department of Physics, School of Physical Sciences, University of Surrey, \\ Guildford, Surrey, GU2 5XH, United Kingdom
}

(Dated: November 5, 2011)

\begin{abstract}
Theories of reactions of composite nuclei simplify considerably at energies of several 100 $\mathrm{MeV} /$ nucleon. Here Glauber methods provide a quantitative microscopic framework with a clear delineation of nucleon-nucleon scattering and nuclear structure inputs. However further approximations, tested for stable nuclei, are inappropriate for few-body halo nuclei with implications for analyses of both total reaction and elastic scattering cross sections. At lower projectile energies, of order tens of $\mathrm{MeV} /$ nucleon, reactions are more usefully formulated in terms of the optical interactions of the projectile constituents and the target, however corrections to Glauber theory are now large. A framework for improving such calculations at lower energies is also presented.
\end{abstract}

PACS numbers: PACS numbers: 24.10.-i, 24.10.Eq, 24.50.+g, 25.10.+s 


\section{FEW-BODY CALCULATIONS AT HIGH ENERGY}

Neutron dripline nuclei have very weak binding of the last neutron(s). There is therefore a very large amplitude for finding these valence nucleons in the classically-forbidden region beyond a tightly bound core - producing well developed few-body structures. The root mean squared (rms) matter radii of such nuclei are therefore large, manifest empirically as large interaction/reaction cross sections with a target probe. Such data remain the clearest experimental signature of these novel structures [1], however new data, on elastic scattering of neutron-rich light nuclei from both proton and more massive targets, also demonstrate significant sensitivity to the halo size, e.g. [2, 3].

Good examples are the experimental data of the IKAR collaboration [4] on $p+{ }^{6} \mathrm{He}$ and ${ }^{8} \mathrm{He}$ scattering near $700 \mathrm{MeV}$, shown in Figure 1 as a function of the momentum transfer squared, $q^{2}=-t$. The data have been scaled, as indicated, to coincide at small $q^{2}$ and with the available $p+\alpha$ data. The lines are to guide the eye. Qualitatively, the increasing size of the He isotopes with $A$ is clear, but data for a stable nucleus of known size, such as ${ }^{6} \mathrm{Li}$, would help provide a scale for comparison with the neutron-rich systems. The rms size of ${ }^{6} \mathrm{He}$ deduced from these data in ref. [4] (2.30土0.07 fm) is less than one would expect. It suggests ${ }^{6} \mathrm{He}$ is actually smaller than ${ }^{6} \mathrm{Li}$ (with deduced matter radius of $2.44 \mathrm{fm}$ from electron scattering [5]), whereas interaction cross section measurements at $800 \mathrm{MeV} /$ nucleon yield a cross section for ${ }^{6} \mathrm{He}+{ }^{12} \mathrm{C}[6](722 \pm 5 \mathrm{mb})$ significantly greater than for ${ }^{6} \mathrm{Li}+{ }^{12} \mathrm{C}[7]$ $(688 \pm 10 \mathrm{mb})$.

It was already shown, in total reaction cross section calculations [8-10], that an explicit treatment of the few-body degrees of freedom of such light nuclei is of considerable quantitative importance. These few-body effects were shown to increase the transparency of the elastic $S$-matrix $\mathcal{S}_{A}(b)$ at large impact parameters $b[9]$ leading to smaller calculated reaction cross sections - and hence to larger deduced nuclear sizes from comparisons with data. Whereas a matter radius for ${ }^{6} \mathrm{He}$ consistent with a simplified (one-body density based) analysis was $2.33 \mathrm{fm}$ [1], the value $2.54 \mathrm{fm}$ is consistent with a more careful few-body analysis [10] of the same experimental datum.

Since, in a projectile-target (of mass $A$ ) collision, the reaction cross section

$$
\sigma_{R}(A)=2 \pi \int_{0}^{\infty} d b b\left[1-\left|\mathcal{S}_{A}(b)\right|^{2}\right]
$$


and the elastic scattering amplitude, at momentum transfer $q$,

$$
f_{A}(q)=i k \int_{0}^{\infty} d b b J_{0}(q b)\left[1-\mathcal{S}_{A}(b)\right]
$$

are both integrals involving $\mathcal{S}_{A}(b)$ and different weight functions, analogous few-body corrections are anticipated in elastic scattering. Here $k$ is the projectile's incident wave number in the centre of mass (c.m.) frame. We discuss briefly a first treatment of these few-body degrees of freedom in calculations of $p+{ }^{A} \mathrm{He}$ scattering at energies near $700 \mathrm{MeV}$, and their implications for the sizes of the He isotopes deduced from the experimental data [4].

\section{A. Few-body methodology}

According to Glauber's multiple scattering theory [11], the elastic amplitude for $p+A$ scattering is given by Eq. (2) where the elastic $S$-matrix at c.m. impact parameter $b$, is

$$
\mathcal{S}_{A}(b)=\left\langle\Phi_{A}\left|\prod_{j=1}^{A} S_{j}\left(b_{j}\right)\right| \Phi_{A}\right\rangle .
$$

Here $j$ labels the target nucleons with ground state many-body wavefunction $\Phi_{A}$, but, as the proton scattering experiments of ref. [4] were performed in inverse kinematics, $\left|\Phi_{A}\right\rangle$ is the projectile ground state. The $S_{j}\left(b_{j}\right)=1-\Gamma_{p j}\left(b_{j}\right)$ are the pairwise nucleon-nucleon scattering operators, $b_{j}$ is the impact parameter of the incident proton relative to target nucleon $j$. Index $j$ also identifies the use of the $p n$ or $p p$ profile function, $\Gamma_{p j}$. These are parameterised, as is usual, directly from the free $p p$ and $p n$ scattering data. For details see $[3,12]$.

It must be realised that $\mathcal{S}_{A}(b)$ is a many-body matrix element of the projectile's manybody density $\left|\Phi_{A}\right|^{2}$. It has been common practice however, e.g. [4], based on successful analyses for stable (tightly bound) nuclei $[13,14]$, to replace these many-body densities by products of one-body densities - sometimes putting back c.m. correlations by hand in an approximate way [14]. While for the compact ${ }^{4} \mathrm{He}$ system this is not unreasonable, and is used here, the particular spatial correlations of the nucleons in ${ }^{6} \mathrm{He}$ and ${ }^{8} \mathrm{He}$, into a $T=0$ $\alpha$ core and a neutron halo/skin component, makes such an (uncorrelated) factorisation an uncertain and unquantified procedure.

For halo nuclei, with their well developed few-body structures, an alternative $n$-cluster description is appropriate. Due to the weak valence nucleon binding, the expectation is also that core polarisation effects are small, particularly for the He isotopes [15]. The many-body 
wavefunction is then a product of an intrinsic wavefunction $\Phi_{A_{c}}$ for the mass $A_{c}$ core and an $n$-body wavefunction $\psi_{\text {rel }}^{(n)}$ describing the relative motion of all clusters. The $n$-cluster variant of the $A$-body $S$-matrix element is thus

$$
\mathcal{S}_{A}^{(n)}(b)=\left\langle\psi_{\text {rel }}^{(n)}\left|\mathcal{S}_{A_{c}}\left(b_{c}\right) \prod_{j=1}^{n-1} S_{j}\left(b_{j}\right)\right| \psi_{\text {rel }}^{(n)}\right\rangle,
$$

where $\mathcal{S}_{A_{c}}$ is the free $p+$ core elastic $S$-matrix at the same incident energy per nucleon, given by Eq. (3) with $A=A_{c}$.

\section{B. Results for $p+{ }^{A}$ He scattering}

Within this few-body model the scattering of the $n$-cluster nucleus is seen to be predicted, without free parameters, given $(i)$ the scattering of the constituents, and $(i i)$ their relative motion wave function $\psi_{\text {rel }}^{(n)}$. Critically however $\mathcal{S}_{A}^{(n)}$ remains a many-body matrix element, now of the projectile's few-body density $\left|\psi_{\text {rel }}^{(n)}\right|^{2}$, and, in general, has no simple relationship to the projectile's one-body density.

The calculated $p+\alpha S$-matrix, $\mathcal{S}_{4}$, using a simple (c.m. correlated) $(0 s)^{4}$ oscillator fourbody $\alpha$ density

$$
\left|\Phi_{4}\left(\vec{r}_{1}, \vec{r}_{2}, \vec{r}_{3}, \vec{r}_{4}\right)\right|^{2} \propto \prod_{j=1}^{4}\left|\phi\left(r_{j}\right)\right|^{2} \delta\left(\vec{r}_{1}+\vec{r}_{2}+\vec{r}_{3}+\vec{r}_{4}\right)
$$

reproduces the $699 \mathrm{MeV} p+\alpha$ data of Figure 1 [3] for an $\alpha$ rms matter radius of $1.49 \mathrm{fm}$, consistent with electron scattering [5], and is not shown. To calculate $\mathcal{S}_{4}$ the $S_{j}$ used is the $(T=0)$ average of the $p n$ and $p p$ amplitudes. Given $\mathcal{S}_{4}$ and $\Gamma_{p n}$ all inputs to the ${ }^{6} \mathrm{He}$ and ${ }^{8} \mathrm{He}$ calculations, other than the relative motion wavefunctions $\psi_{\text {rel }}^{(n)}$, are completely determined.

Figure 2 shows the predicted $p+{ }^{6} \mathrm{He}$ elastic cross sections at $717 \mathrm{MeV}$ using three $(\mathrm{P} 1$, FC and GB3) Faddeev wavefunction models $\psi_{\text {rel }}^{(3)}$ tabulated in [9]. These yield ${ }^{6}$ He radii of 2.33, 2.50, and $2.77 \mathrm{fm}$, respectively, assuming an $\alpha$ rms radius of $1.49 \mathrm{fm}$. The figure shows that the elastic scattering data are consistent with the FC-model wavefunction with rms radius $2.50 \mathrm{fm}$. The data are subject to a normalisation uncertainty of order $\pm 3 \%$ [4]. The inset shows the calculated total reaction cross sections as a function of the ${ }^{6} \mathrm{He}$ rms matter radius for several wavefunction models. These show significant sensitivity and, if accessible experimentally, could provide a powerful constraint in combination with the $q^{2}$ data. 
The approximate (one-body density) analysis of [4] and the few-body analysis above lead to quite different outcomes. While the density-based calculations suggest a radius of 2.30 $\mathrm{fm}$ is appropriate [4], the more careful few-body treatment of the reaction suggests a radius of order $2.50 \mathrm{fm}$. We comment that the FC wavefunction also reproduces the experimental ${ }^{6} \mathrm{He}+{ }^{12} \mathrm{C}$ interaction cross section datum at $800 \mathrm{MeV} /$ nucleon in the careful finite range study of that process [10]. The FC model also reproduces most closely the empirical ${ }^{6} \mathrm{He}$ three-body binding energy of $0.97 \mathrm{MeV}$.

Five-body $(\alpha+4 n)$ descriptions of ${ }^{8} \mathrm{He}$ are less readily available. Here we assume for $\psi_{\text {rel }}^{(5)}$ the COSMA wavefunction of [16], however the original $(0 p)^{4}$ oscillator valence neutron wavefunctions are now matched smoothly to a $p$-wave Hankel function tail. As the twoneutron separation energy from ${ }^{8} \mathrm{He}$ is $2.137 \mathrm{MeV}$ and the four-neutron separation energy is $3.1 \mathrm{MeV}$, we assume an average separation energy of $1 \mathrm{MeV}$ for this purpose. The wavefunction is then renormalised to unity and the ${ }^{8} \mathrm{He}$ matter radius of is computed.

Our first calculations for this system are collected in Figure 3 which shows the predicted $p+{ }^{8} \mathrm{He}$ elastic cross section at $674 \mathrm{MeV}$. The calculations correspond to the ${ }^{8} \mathrm{He}$ rms matter radii indicated. Calculations for radii in the range $2.4-2.5 \mathrm{fm}$, suggested by the analysis of [4], do not reproduce the experimental data. A radius of $2.6 \mathrm{fm}$ is consistent with the data within the COSMA model used. Again the inset shows the calculated total reaction cross sections as a function of the ${ }^{8} \mathrm{He}$ rms matter radius. As for ${ }^{6} \mathrm{He}$ these reveal a significant sensitivity to the projectile size.

We observe significant sensitivity in the calculated cross section to the wavefunction asymptotics and conclude, quite generally, that a careful and realistic treatment of these few-body systems will be essential to making quantitative deductions from comparisons with such data.

\section{FEW-BODY CALCULATIONS AT LOW ENERGY}

We now consider calculations for few-body projectiles at energies of a few 10's of MeV per nucleon. At these lower energies the reaction mechanisms are more complex and the interactions in the two-body subsystems are best deduced, as far as possible, from empirical data and established theoretical models for stable nuclei. Recent calculations for ${ }^{8} \mathrm{He}+{ }^{12} \mathrm{C}$ scattering [17], as well as those above for $p+{ }^{8}$ He scattering - treated as six-body problems 
- show that the Glauber models provide an effective basis for the calculation of reactions of few- and many-body projectiles. This efficiency arises from the very simple (independent scattering) nature of each two-body input. This efficiency warrants an investigation of corrections to the model in an attempt to extend its accuracy.

${ }^{11} \mathrm{Be}$ and ${ }^{6} \mathrm{He}$ are good examples of single-neutron and two-neutron halo nuclei. Some elastic scattering data are also available for both systems in the energy region of interest. For two-body projectiles [18], and very recently for three-body projectiles [19], full quantum mechanical calculations, which use the adiabatic approximation but not the Glauber/eikonal and independent scattering assumptions, are possible. These will be used to assess the nature of corrections to the lowest order theory.

For an $n$-body projectile scattering from a target nucleus, rather than a proton, the elastic amplitude is given, as previously, by Eqs. (2) and (3) but where now the $S_{j}$ are the Glauber $S$-matrices for each constituent $j$-target subsystem, interacting via a potential $V_{j}$. Explicitly [11]

$$
S_{j}(b)=\exp \left[i \chi_{j}^{0}(b)\right], \quad \chi_{j}^{0}(b)=-\frac{1}{\hbar v} \int_{-\infty}^{\infty} V_{j}\left(\sqrt{b^{2}+z^{2}}\right) d z
$$

where $v$ is the asymptotic relative velocity and the $z$-axis is in the incident beam direction. $\chi_{j}^{0}$ is referred to as the eikonal phase.

\section{A. Beyond the eikonal model}

Following Wallace [20] and others, we have recently made use of the correspondence between the eikonal phase and the WKB phase shift $\chi_{j}^{W}$. The latter can be expanded, in powers of $\epsilon=1 / \hbar k v$, about the eikonal phase [21]

$$
\chi_{j}^{W}(b)=\sum_{n=0}^{\infty} \frac{\epsilon^{n}}{(n+1) !} \chi_{j}^{n}(b), \quad \chi_{j}^{n}(b)=-\frac{1}{\hbar v} \int_{-\infty}^{\infty} d z\left(\frac{1}{r} \frac{d}{d r}\right)^{n}\left[r^{2 n} V_{j}(r)^{n+1}\right],
$$

which is the $n=0$ term. The $\chi_{j}^{W}$ require correction terms $\chi_{j}^{R Y}$, as clarified by Rosen and Yennie [22].

We have shown [23] that improving the phase in each $S_{j}$ of the few-body model, by replacing the eikonal phase $\chi_{j}^{0}$ by $\chi_{j}^{W}+\chi_{j}^{R Y}$, leads to significant changes and improvements in calculations, when compared with exact adiabatic results. Doing this retains the independent scattering nature of the lowest order theory and so neglects "overlapping potential" terms 
[24]. The indications from our calculations [23] are that such terms, from configurations in which both core and valence particles overlap the target, are very small for extended halo nuclei with a strongly absorbed core particle.

Having established that the non-eikonal corrections are large, one finds that the expansions involved in calculating the constituent $\chi_{j}^{W}+\chi_{j}^{R Y}$ converge only very slowly to the exact (partial wave) phases as the projectile energy is reduced. Figure 4 shows $\left|S_{n}(b)\right|$, for $n+{ }^{12} \mathrm{C}$ at $25 \mathrm{MeV}$, calculated in the eikonal model (dot-dashed), the WKB and RY corrected model (dashed), up to $n=3$ terms, and the exact partial wave values (points and solid line) where $k b=\ell+\frac{1}{2}$. Rather than sum this expansion we therefore propose to solve the radial Schrödinger equation, for each constituent $j$, at the required impact parameters $b$, and hence non-integer angular momenta $\lambda=b k_{j}-\frac{1}{2}$. The $S_{j}(b)$ are then obtained by matching, in the normal manner, to the asymptotic solutions, analytically continued for real non-integer $\lambda$, i.e.

$$
\psi_{\lambda}^{j}(R) \rightarrow \frac{i}{2}\left[H_{\lambda}^{(-)}\left(k_{j} R\right)-S_{j}(b) H_{\lambda}^{(+)}\left(k_{j} R\right)\right] .
$$

We refer to this procedure as an impact parameter multiple scattering (IPMS) approximation. The differences between few-body calculations using these independent $S_{j}$ and exact (non-eikonal) adiabatic calculations provide a measure the importance of the neglected terms due to overlapping potentials, or correlated scattering.

\section{B. Comparisons of eikonal and non-eikonal calculations}

Figure 5 shows calculated elastic differential cross sections (ratio to Rutherford) for ${ }^{11} \mathrm{Be}+{ }^{12} \mathrm{C}$ scattering at 50, 25 and $10 \mathrm{MeV} /$ nucleon for selected two-body interactions $V_{j}$ [23]. The dot-dashed curves show the results of the lowest order eikonal model calculation. The IPMS results are shown by the long-dashed curves which are seen to agree to high precision, at even the lowest energy, with the exact three-body adiabatic model calculations [18], shown by the solid curves. Figure 6 shows analogous elastic differential cross section (ratio to Rutherford) calculations for ${ }^{6} \mathrm{He}+{ }^{12} \mathrm{C}$ scattering at 41.6 and $25 \mathrm{MeV} /$ nucleon. Again the dot-dashed curves are the results of the lowest order eikonal model calculations. The IPMS results are shown by the long-dashed curves and the exact four-body adiabatic model calculations [19] by the solid curves. The IPMS calculations are performed in a small fraction of the time of the coupled channels adiabatic solutions and are readily generalised to 
many-body systems.

We conclude that the IPMS framework provides an effective procedure to enhance the accuracy of few-body Glauber model calculations at low energies for one and two-neutron halo nuclei. It remains to be tested, for a neutron skin nucleus such as ${ }^{8} \mathrm{He}$ with several valence neutrons in a more confined volume, whether this independent scattering picture remains as accurate.

\section{SUMMARY}

We have discussed briefly the need for quantitative calculations of few-body scattering and reactions at energies from 10 's $\mathrm{MeV}$ to $1 \mathrm{GeV} /$ nucleon, to confront experimental data of increased novelty and precision. We have shown that, within a few-body model of ${ }^{6} \mathrm{He}$, the recent $p+{ }^{6} \mathrm{He}$ elastic scattering data at $700 \mathrm{MeV}$ are consistent with the same three-body

${ }^{6} \mathrm{He}$ wavefunction that reproduces the interaction cross section measurement for ${ }^{6} \mathrm{He}+{ }^{12} \mathrm{C}$ at $800 \mathrm{MeV} /$ nucleon. The deduced ${ }^{6} \mathrm{He}$ and ${ }^{8} \mathrm{He}$ sizes from the few-body calculations are $0.2 \mathrm{fm}$ larger than those reported from the more approximate analysis of ref. [4].

We have also shown that, in the adiabatic approximation, even at very low energies, to high accuracy halo nuclei behave as if the constituent particles scatter independently, suggesting a simple practical framework for increasing their accuracy. This is shown to work well for one- and two-neutron halo nuclei.

\section{Acknowledgments}

The financial support of the United Kingdom Engineering and Physical Sciences Research Council (EPSRC) in the form of Grant No. GR/J95867, of a research studentship (for JMB), and of a Royal Society Travel Grant are gratefully acknowledged.

[1] I. Tanihata et al., Phys. Lett. B160 (1985) 380; B206 (1988) 592.

[2] R.C. Johnson, J.S. Al-Khalili and J.A. Tostevin, Phys. Rev. Lett. 79 (1997) 2771.

[3] J.S. Al-Khalili, J.A. Tostevin, Few-body calculations of $p^{-6,8}$ He scattering, submitted 11/97.

[4] G.D. Alkhazov et al., Phys. Rev. Lett. 78, (1997) 2313. 
[5] H. de Vries, C.W. de Jager and C. de Vries, At. Data Nucl. Data Tables 36 (1987) 495.

[6] I. Tanihata, D. Hirata, T. Kobayashi, S. Shimoura, K. Sugimoto and H. Toki, Phys. Lett. B289 (1992) 261.

[7] I. Tanihata et al., Phys. Rev. Lett. 55 (1985) 267.

[8] J.S. Al-Khalili and J.A. Tostevin, Phys. Rev. Lett. 76 (1996) 3903.

[9] J.S. Al-Khalili, J.A. Tostevin and I.J. Thompson, Phys. Rev.C 54 (1996) 1843.

[10] J.A. Tostevin and J.S. Al-Khalili, Nucl. Phys. A616 (1997) 418c.

[11] R.J. Glauber, Lectures in Theoretical Physics, (Interscience, N.Y., 1959) Vol. 1, 315.

[12] L. Ray, Phys. Rev. C 20 (1979) 1857.

[13] S. Kox et al., Phys. Rev. C 35 (1987) 1678.

[14] G.D. Alkhazov, S.L. Belostotsky and A.A. Vorobyov, Phys. Rep. 42C (1978) 89.

[15] T.T.S. Kuo, F. Krmpotić, and Y. Tzeng, Phys. Rev. Lett. 78 (1997) 2708.

[16] M.V. Zhukov, A.A. Korsheninnikov and M.H. Smedberg, Phys. Rev. C 50 (1994) R1.

[17] J.A. Tostevin, J.S. Al-Khalili, M. Zahar, M. Belbot, J.J. Kolata, K. Lamkin, D.J. Morrissey, B.M. Sherrill, M. Lewitowicz, and A.H. Wuosmaa, Phys. Rev. C 56 (1997) 2929R.

[18] H. Amakawa, S. Yamaji, A. Mori and K. Yazaki, Phys. Lett. 82B (1979) 13.

[19] J.A. Christley, J.S. Al-Khalili, J.A. Tostevin, R.C. Johnson, Nucl. Phys. A624 (1997) 275.

[20] S.J. Wallace, Phys. Rev. D 8 (1973) 1846.

[21] S.J. Wallace, Ann. Phys. (N.Y.) 78 (1973) 190; Phys. Rev. Lett. 27 (1971) 622.

[22] M. Rosen and D.R. Yennie, J. Math. Phys. 5 (1964) 1505.

[23] J.S. Al-Khalili, J.A. Tostevin and J.M. Brooke, Phys. Rev. C 55 (1997) R1018.

[24] H. Feshbach, Theoretical Nuclear Physics: Nuclear Reactions, (Wiley, N.Y., 1992), 103; and references therein. 


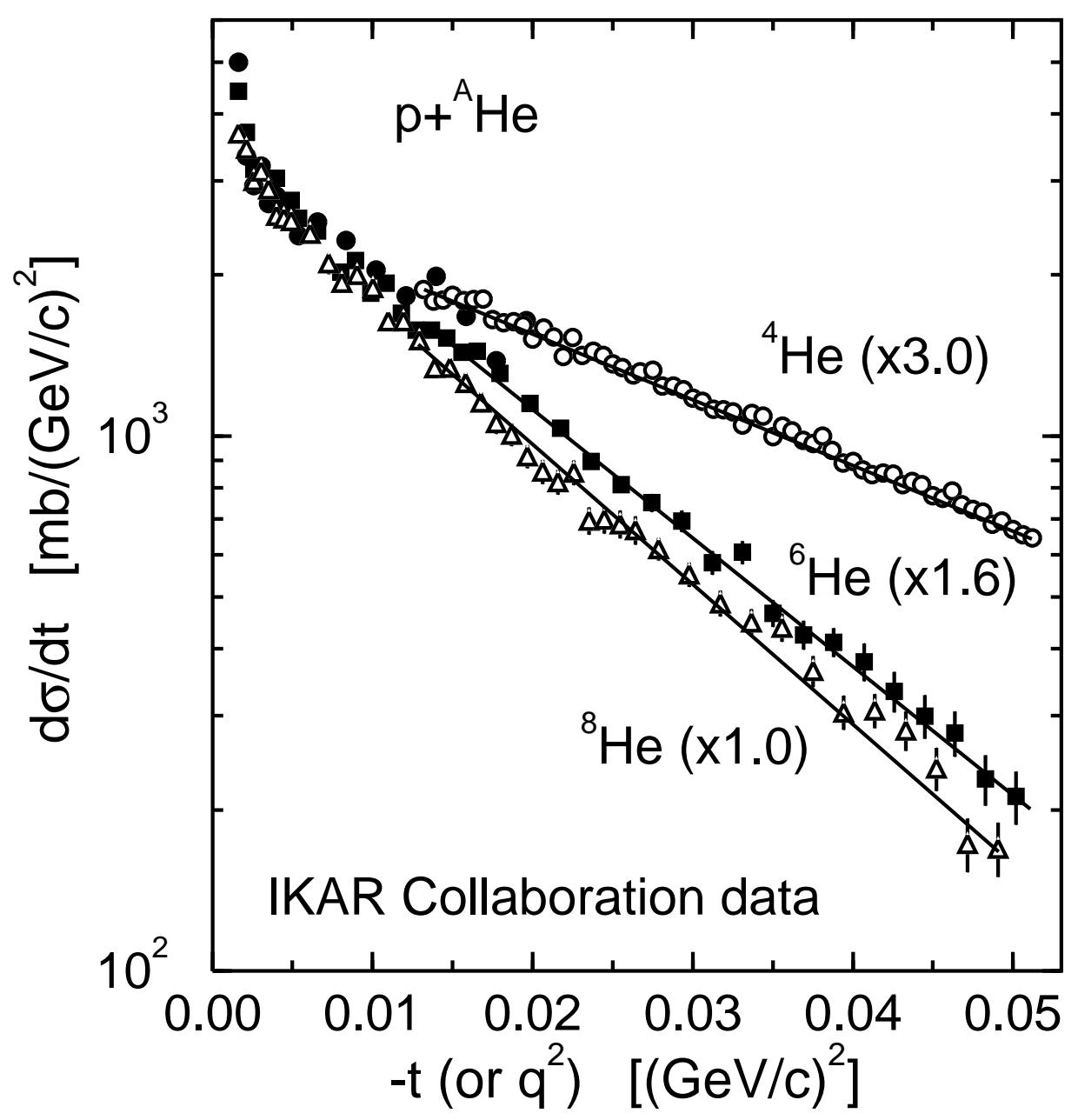

FIG. 1: FIG.1. $p+{ }^{4} \mathrm{He},{ }^{6} \mathrm{He}$, and ${ }^{8} \mathrm{He}$ data at 699,717 , and $674 \mathrm{MeV}$, respectively.

Figures 


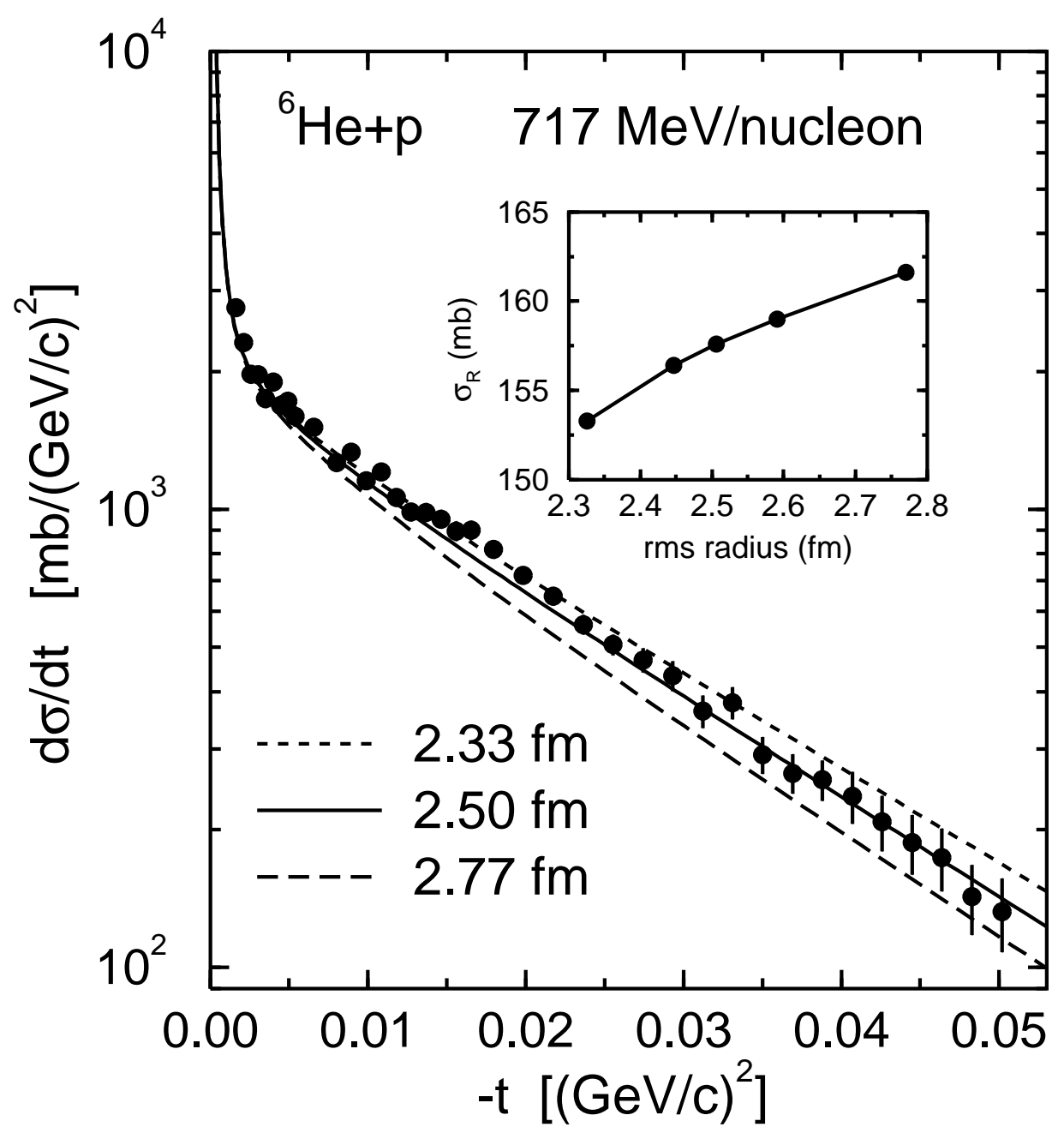

FIG. 2: FIG.2. $p+{ }^{6} \mathrm{He}$ elastic differential cross sections at $717 \mathrm{MeV}$. 


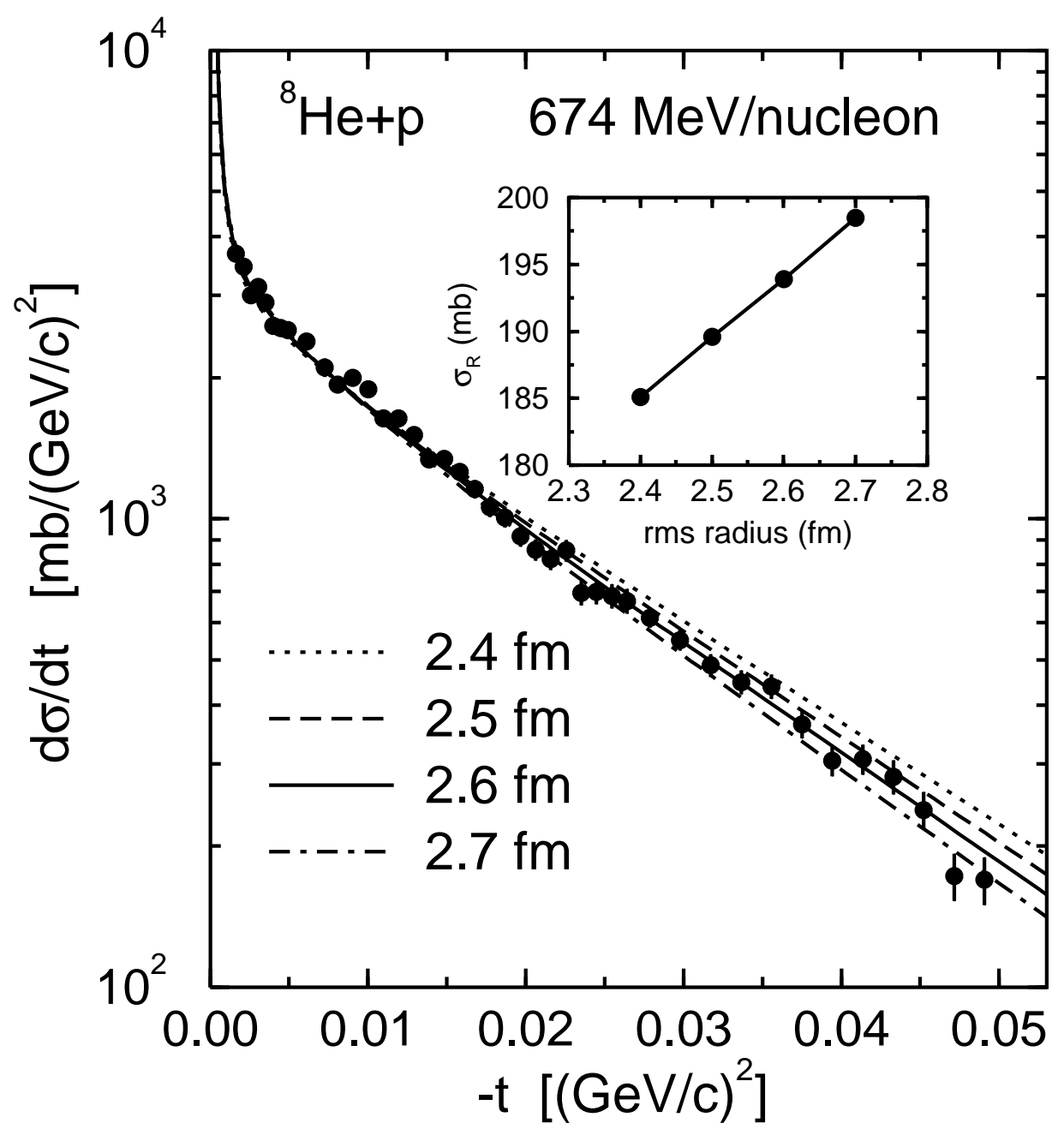

FIG. 3: FIG.3. $p+{ }^{8} \mathrm{He}$ elastic differential cross sections at $674 \mathrm{MeV}$. 


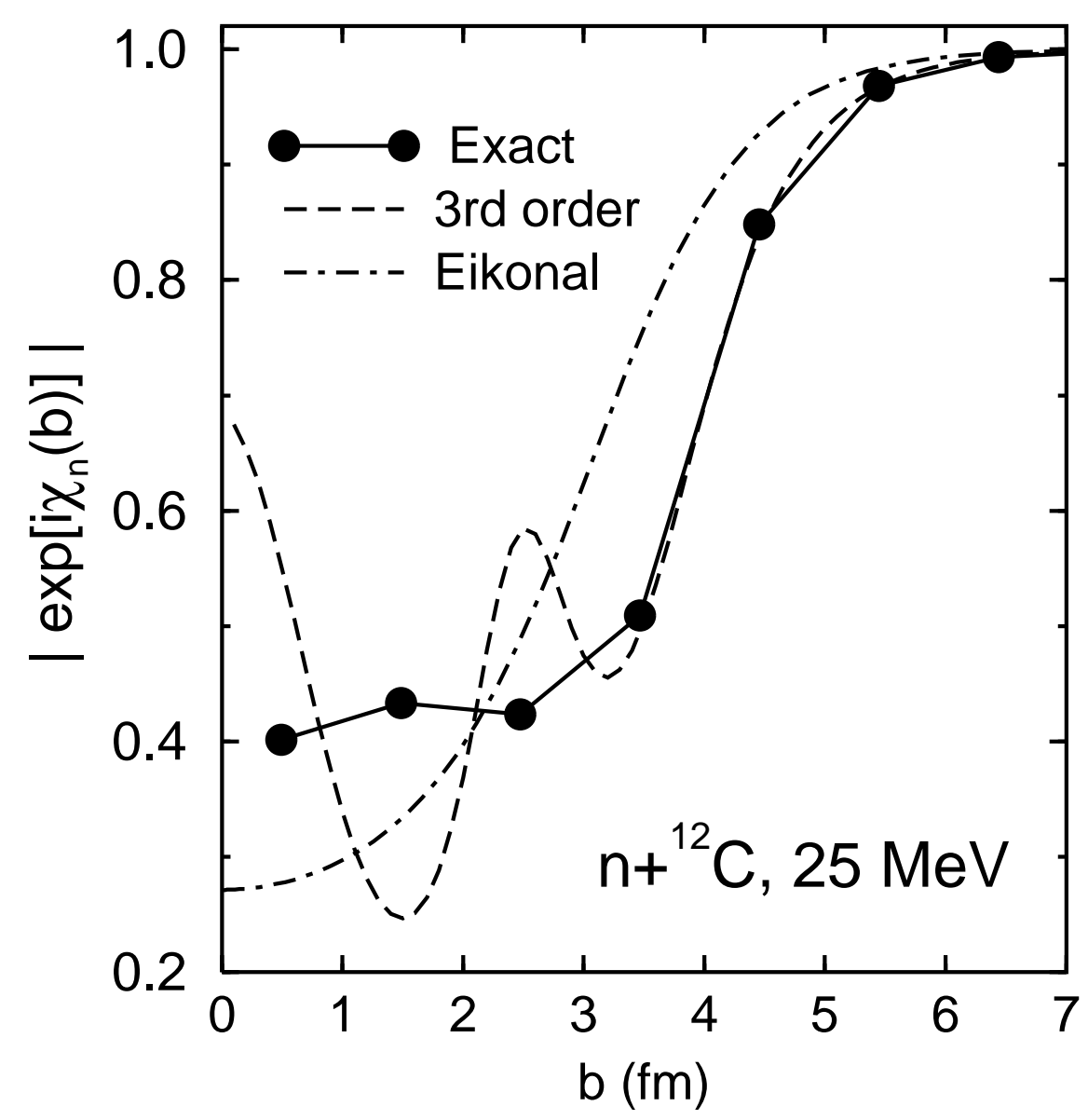

FIG. 4: FIG.4. Modulus of the $n+{ }^{12} \mathrm{C} S$-matrix at $25 \mathrm{MeV}$. 


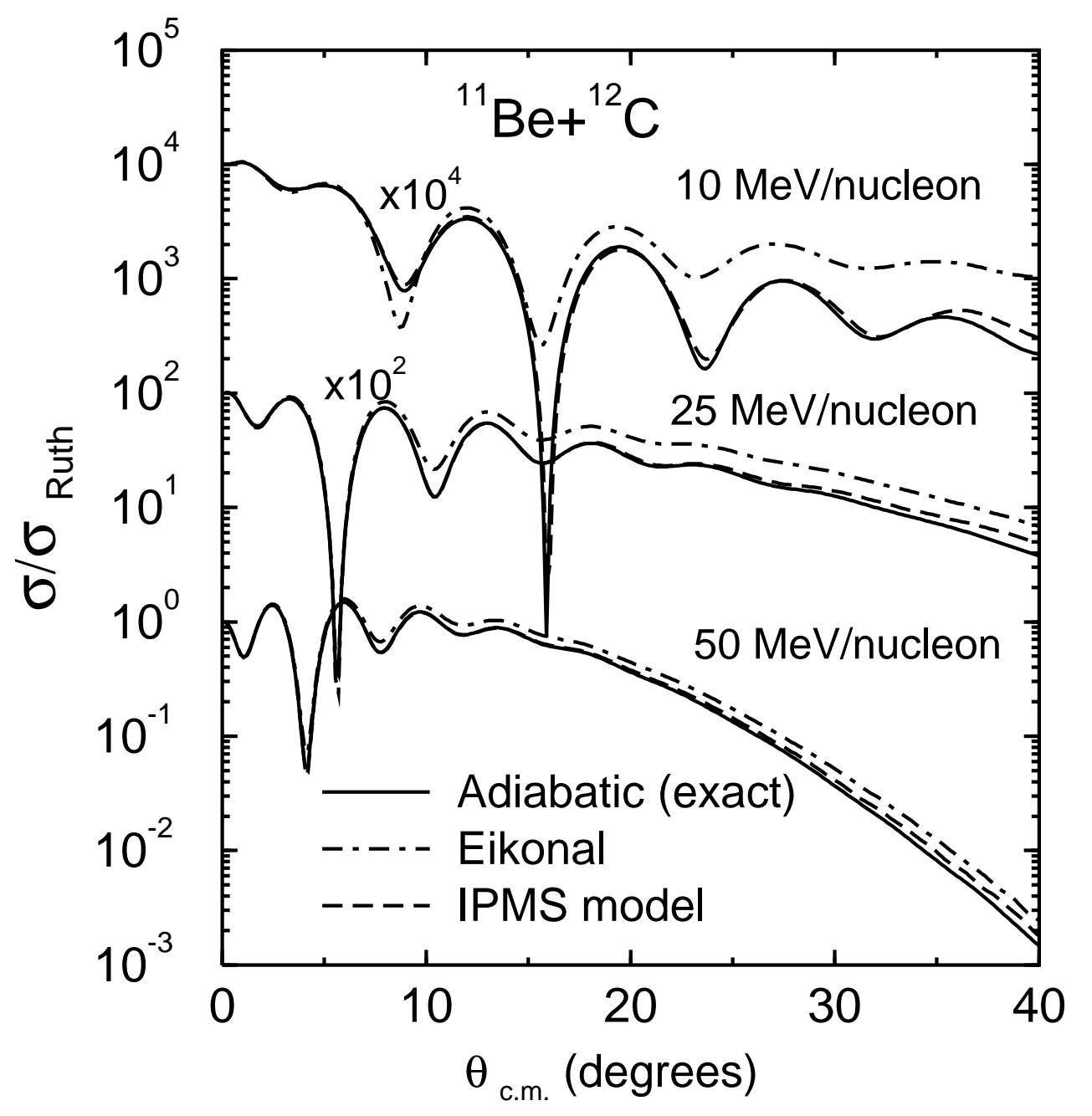

FIG. 5: FIG.5. Elastic cross sections for ${ }^{11} \mathrm{Be}+{ }^{12} \mathrm{C}$. 


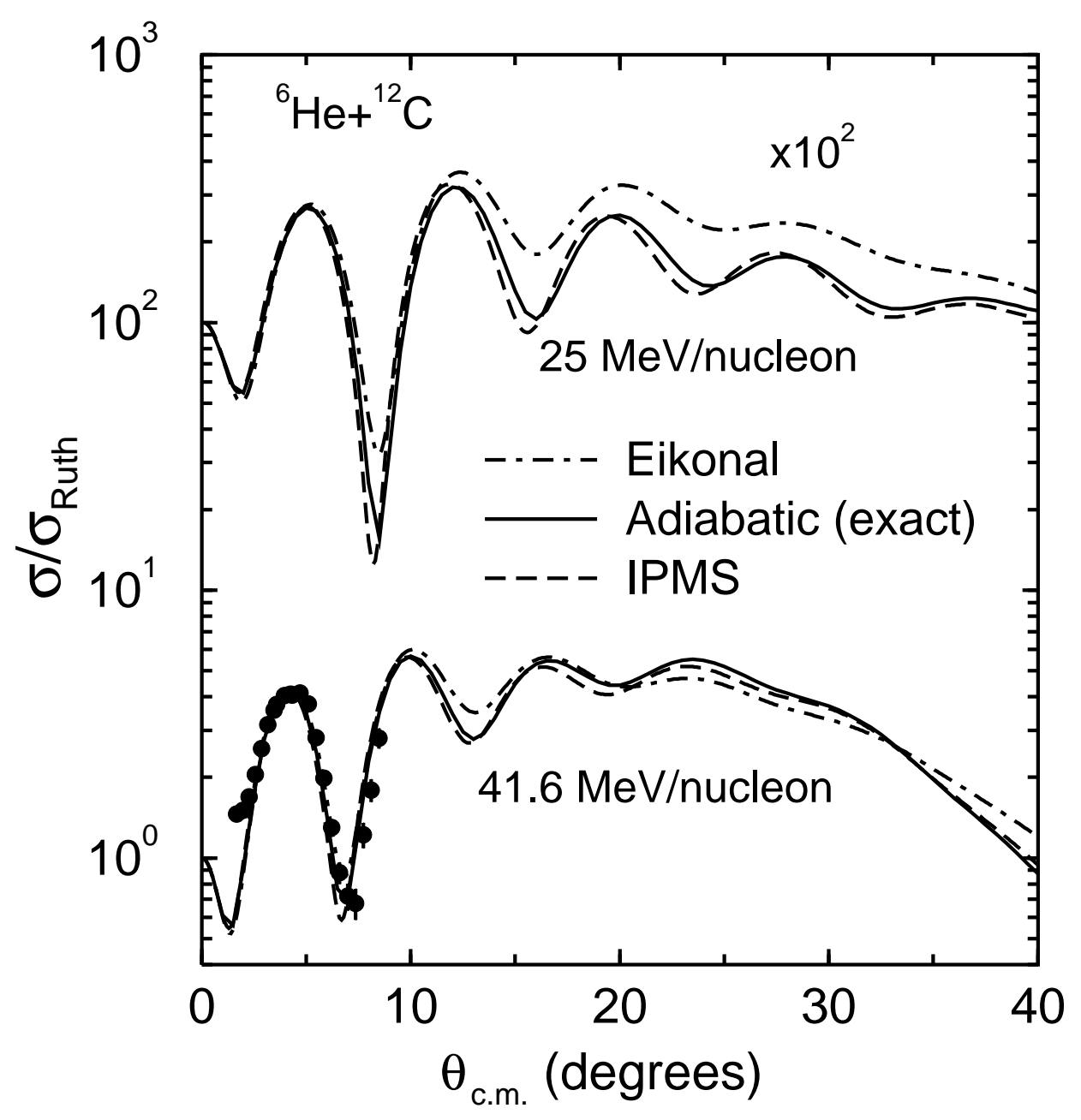

FIG. 6: FIG.6. Elastic cross sections for ${ }^{6} \mathrm{He}+{ }^{12} \mathrm{C}$. 